\title{
Erratum to: chTLR4 pathway activation by Astragalus polysaccharide in bursa of Fabricius
}

Ruili Zhang ${ }^{1}$, Qun Yu ${ }^{1,2}$, Guangliang Shi ${ }^{1}$, Rui Liu', Weiqian Zhang ${ }^{1}$, Xia Zhao ${ }^{1}$, Guangxing Li $^{1}$ and Ming Ge ${ }^{1 *}$

\section{Erratum}

The original article [1] contains an error whereby the authors' given \& family names were mistakenly interchanged.

The original article has now been corrected to present the authors' names correctly.

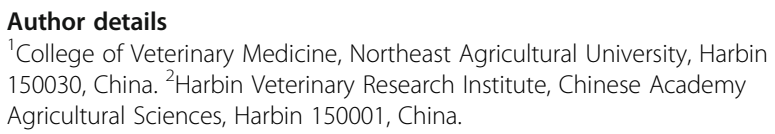

\section{Reference}

1. Zhang R, et al. chTLR4 pathway activation by Astragalus polysaccharide in bursa of Fabricius. BMC Vet Res. 2017;13:119.

\footnotetext{
* Correspondence: gemingneau@163.com

'College of Veterinary Medicine, Northeast Agricultural University, Harbin 150030, China
} 